Polymer Journal Vol. 1, No. 5, pp 493-498 (1970)

\title{
Alternating Copolymers from trans-1, 2-Dibenzoylethylene and trans-1, 2-Diacetylethylene
}

\author{
Toshikazu Nishimura, Tadayuki Yogo, Chiaki Azuma, \\ and Naoya OGAtA \\ Department of Chemistry, Sophia University, Chiyoda-ku, \\ Tokyo, Japan.
}

(Received February 3, 1970)

\begin{abstract}
Symmetrical $\alpha, \beta$-unsaturated carbonyl compounds, trans-1, 2-dibenzoylethylene (DBE) and trans-1, 2-diacetylethylene (DAE) were synthesized and copolymerization with styrene or $n$-butyl vinyl ether was carried out in the presence of a radical initiator. The apparent over-all rates of the copolymerization of DBE were faster than that of DAE and the addition reaction of DBE with amine was also faster than that of DAE, indicating that the double bond of DBE is more electron-deficient than DAE.

The compositions of copolymers from DBE or DAE were constant at the molar ratio of $50 \mathrm{~mol} \%$, regardless of the monomer ratios in the feed, although DAE did not copolymerize with $n$-butyl vinyl ether. No evidence could be found from spectroscopic studies for the formation of a charge-transfer complex between DBE or DAE and donor monomers, and the alternating tendency of the copolymerization could be increased by the strongly favorable polar effect of two carbonyl groups on the double bond of DBE or DAE.

Neither DBE nor DAE copolymerized with styrene with an anionic initiator, possibly because they formed anions too stable to initiate copolymerization.

KEY WORDS Alternating Copolymer/trans-1, 2-Dibenzoylethylene / trans-1,2-Diacetylethylene / Styrene / $n$-Butyl Vinyl Ether / ChargeTransfer Complex/
\end{abstract}

An $\alpha, \beta$-unsaturated carbonyl compound such as maleic anhydride or maleimide is known to copolymerize with styrene to form a highly regular, alternating copolymer in the presence of a radical initiator. The copolymerization reaction could be aided considerably by the strongly favorable polar effect of carbonyl groups, possibly to the point of the two monomers forming a charge-transfer complex.

It was previously found ${ }^{1}$ that the radical copolymerization of trans-1, 2-dibenzoylethylene, which is a fully conjugated system, yielded an alternating copolymer with styrene. A substituent on the phenyl group of trans-1,2dibenzoylethylene, whether electron-donating or accepting, had no effect on copolymer composition and the polar effect of the carbonyl group was found to play an important role in the alternating tendency.

It is interesting to study the copolymerization behavior of trans-1, 2-diacetylethylene since the electron-donating effect of two methyl groups may reduce the electron-deficient character of the carbon-carbon double bond, compared with trans-1,2-dibenzoylethylene, and hence may reduce the alternating tendency. In this study the copolymerization behavior of trans-1,2dibenzoylethylene with styrene or $n$-butyl vinyl ether has been compared with that of trans1,2-diacetylethylene and the role of the carbonyl groups in the copolymerization has been discussed.

\section{EXPERIMENTAL}

Synthesis of trans-1, 2-Dibenzoylethylene (DBE) and trans-1, 2-Diacetylethylene (DAE)

DBE was synthesized by the Friedel-Crafts reaction of fumaroyl chloride with benzene as follows. ${ }^{2}$ 


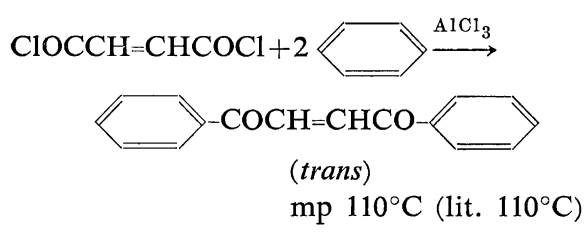

DAE was synthesized by the Wittig reaction as shown in the following routes. ${ }^{3-5}$

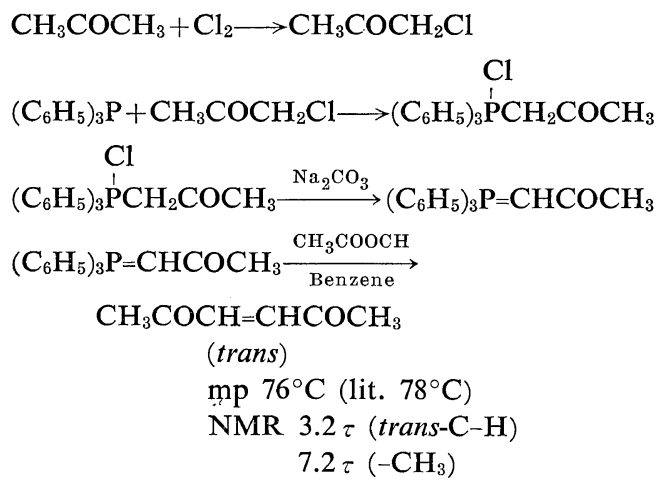

\section{Copolymerization}

The copolymerization was carried out in solution at various molar ratios of DBE or DAE together with styrene of $n$-butyl vinyl ether in the presence of $1 \mathrm{~mol} \%$ of azobisisobutyronitrile as an initiator. The monomer solution was heated in sealed tubes in vacuo for a given period.

The rate of the copolymerization of styrene or $n$-butyl vinyl ether was measured by gas chromatographic analysis using a PEG column at $70^{\circ} \mathrm{C}$. Since $\mathrm{DBE}$ has a yellow color, the amount of unreacted DBE in the solution could be determined by measuring the optical density at $400 \mathrm{~m} \mu$. For the copolymerization of DAE it was difficult to measure the rate of consumption of DAE either by gas chromatographic or spectroscopic method as DAE is a white crystal with a high boiling point. The rate of copolymerization of DAE was determined by copolymer yield.

The copolymer from DBE and styrene was insoluble in methanol and the copolymer from DBE and $n$-butyl vinyl ether was soluble in methanol and water. The copolymer of DAE with styrene was soluble in methanol and insoluble in ethyl ether. Each copolymer was recovered by evaporating copolymer solutions in vacuo at room temperature in the presence of a small amount of hydroquinone to ensure that homopolymerization or copolymerization did not occur during the concentration process. The residual copolymers were fractionated in a silicagel column using benzene-ethyl ether solvent as an eluent to isolate the copolymers.

\section{Reactivity of $D B E$ and $D A E$}

The reactivity of DBE and DAE towards nucleophilic reagents was determined by the addition reaction of DBE or DAE with $n$ butylamine in tetrahydrofuran, where the rate of the addition reaction was determined by gas chromatographic analysis of the unreacted $n$ butylamine in the solution. ${ }^{6}$<smiles>[R]CC([R])NCCNCCC(=O)OC=CC([R])=O</smiles>
$\mathrm{R}:-\mathrm{CH}_{3},-\mathrm{C}_{6} \mathrm{H}_{5}$

An attempt was made to detect the formation of a charge-transfer complex between DBE or DAE and styrene or $n$-butyl vinyl ether by measuring the ultraviolet spectrum of the mixed solution of the two monomers in chloroform, and by applying the continuous variation method proposed by Job. ${ }^{7}$

\section{Analysis of the Copolymers}

After the copolymers were isolated from the solutions, the copolymer compositions were determined both by elemental analysis and from NMR spectra of the copolymers. The elemental analysis was carried out with a Yanagimoto CHN corder and the NMR spectra were measured with a Varian $60 \mathrm{MC}$ spectrometer.

The solution viscosity of the copolymers was determined in benzene solution at a concentration of $1 \%$ and the molecular weight of the copolymers was measured with a vapor pressure osmometer (Mechlorab model 302). The melting point of the copolymers was determined by differential thermal analysis using a RigakuDenki Thermoflex 8001.

\section{RESULTS}

Neither DBE nor DAE homopolymerized at all even after heating for more than 100 hours in benzene at $60^{\circ} \mathrm{C}$ in the presence of a radical initiator. However, copolymerization reactions with electron-donating vinyl monomers took 


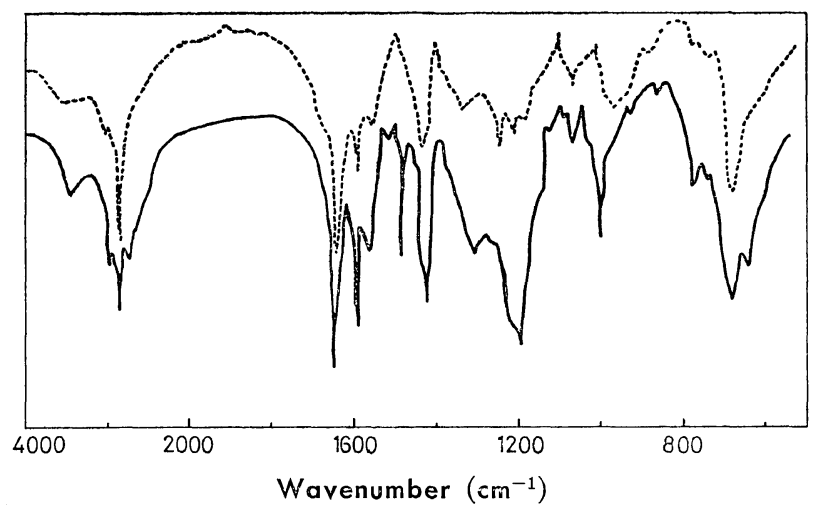

Figure 1. Infrared spectra of the copolymers from DBE with styrene or $n$-butyl vinyl ether: - - with styrene; ------, with $n$-butyl vinyl ether.

place to yield copolymers.

\section{Copolymerization of $D B E$}

The copolymerization of DBE with styrene yielded copolymers having melting points from 200 to $210^{\circ} \mathrm{C}$ and molecular weights of 8000 to 10000 , while the copolymer from DBE and $n$ butyl ether was a grease-like substance without a distinct softening point and its molecular weight was 1500 to 2000 .

The infrared spectra of the copolymers from $\mathrm{DBE}$ and styrene or $n$-butyl vinyl either are shown in Figure 1, where a strong absorption owing to the carbonyl group appears at 1680 $\mathrm{cm}^{-1}$ in both spectra, and absorptions corresponding to monosubstituted benzene or ether groups can be detected. The NMR spectra of the copolymers show proton signals due to phenyl and methylene groups at 3 and $7.5 \tau$, respectively. These results suggest that the copolymerization occured between DBE and styrene or $n$-butyl vinyl ether.

The apparent overall rates of copolymerization at various monomer ratios in the feed are summarized in Table I, where it is seen that DBE copolymerizes with styrene more rapidly than $n$-butyl vinyl ether and the apparent over-all rate of the copolymerization with $n$-butyl vinyl ether is highest at the molar ratio of $50 / 50$ in the feed, while that with styrene decreases with the increasing amount of DBE in the feed.

From the temperature dependence of the apparent over-all rate of the copolymerization, the apparent activation energy for the copolymerization was calculated to be $15.7 \mathrm{kcal} / \mathrm{mol}$

Table I. Apparent over-all rate of copolymerization of DBE $\left(\mathrm{M}_{1}\right)$ with styrene or $n$-butyl viny ether $\left(M_{2}\right)$

\begin{tabular}{|c|c|c|c|c|c|c|}
\hline \multicolumn{2}{|c|}{ Molar ratio } & \multirow{2}{*}{$\mathrm{M}_{2}$} & \multirow{2}{*}{$\begin{array}{l}\text { Monomer } \\
\text { concn, mol/l }\end{array}$} & \multirow{2}{*}{ Temp, ${ }^{\circ} \mathrm{C}$} & \multicolumn{2}{|c|}{ Over-all rate, wt $\% / \mathrm{hr}$} \\
\hline$M_{1}$ & $\mathrm{M}_{2}$ & & & & In dioxane & In benzene \\
\hline 1 & 9 & Styrene & 1 & 60 & 4.2 & 4.5 \\
\hline 3 & 7 & " & " & $" \prime$ & 4.7 & 4.5 \\
\hline 5 & 5 & " & " & " & 4.0 & 3.1 \\
\hline 7 & 3 & " & "I & " & 2.0 & 1.4 \\
\hline 2 & 8 & $n$-Butyl vinyl ether & 0.5 & 70 & - & 0.93 \\
\hline 4 & 6 & " & " & $" 1$ & - & 1.05 \\
\hline 5 & 5 & " & " & " & - & 1.20 \\
\hline 6 & 4 & " & " & " & - & 0.85 \\
\hline 8 & 2 & " & " & " & - & 0.71 \\
\hline
\end{tabular}


for styrene and $11.3 \mathrm{kcal} / \mathrm{mol}$ for $n$-butyl vinyl ether, respectively.

The compositions of the copolymers from DBE and styrene or $n$-butyl vinyl ether, which were determined by elementary anlysis, are shown in Figure 2, where it is seen that the

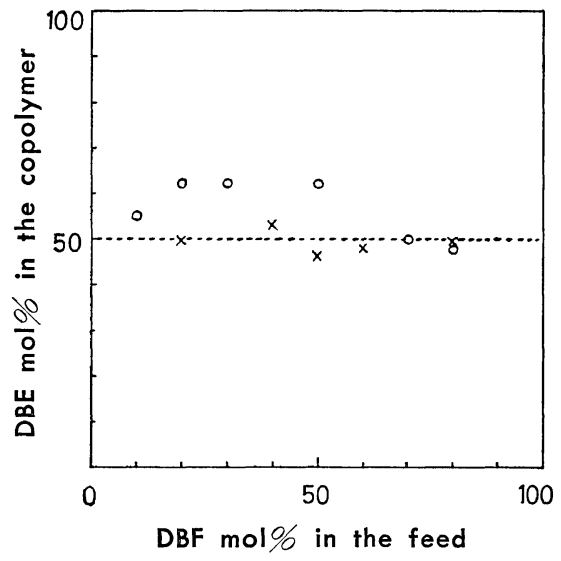

Figure 2. Compositions of the copolymers from DBE with styrene or $n$-butyl ether: $\bigcirc$, with styrene; $\times$, with $n$-butyl vinyl ether.

copolymer compositions are almost constant regardless of the monomer ratios in the feed.

\section{Copolymerization of $D A E$}

It was found that the copolymerization of DAE with styrene yielded a white copolymer with a melting point of 167 to $180^{\circ} \mathrm{C}$ and a molecular weight of 1200 to 1300 , while copolymerization of DAE with $n$-butyl vinyl ether did not occur at all after heating for 10 days at $70^{\circ} \mathrm{C}$.

The infrared spectrum of the copolymer from DAE and styrene is shown in Figure 3, where

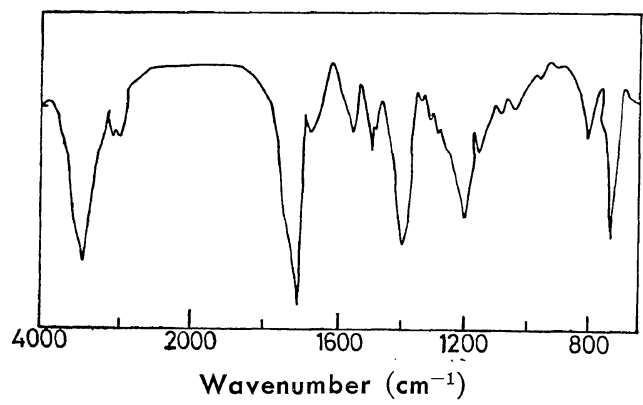

Figure 3. Infrared spectrum of the copolymer from DAE and styrene. an absorption owing to carbonyl group appears at $1720 \mathrm{~cm}^{-1}$ and absorptions owing to monosubstituted benzene appear at 1650 and 760 $\mathrm{cm}^{-1}$. The NMR spectrum of the copolymer exhibits proton signals owing to phenyl groups at 3 and $8.5 \tau$, as shown in Figure 4 . As the

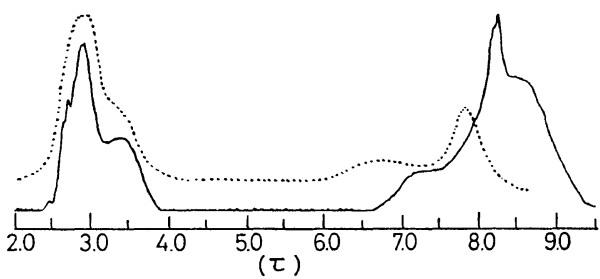

Figure 4. NMR spectrum of the copolymer from DAE or DBE with styrene: - $\longrightarrow$ DAE copolymer; -----, DBE copolymer.

copolymer from DAE and styrene was soluble in methanol, the homopolymer of styrene was easily separated. Therefore, the IR and NMR spectra of the copolymer confirm that the copolymerization reaction took place between DAE and styrene.

The yield of the copolymer increased almost linearly with reaction time up to $16 \mathrm{hr}$ and the apparent over-all rates of the copolymerization at various monomer ratios in the feed are shown in Table II. The apparent over-all rate reaches a maximum at the molar ratio of $50 \mathrm{~mol} \%$ of DAE in the feed. The apparent activation energy for the copolymerization at the molar ratio of $50 \mathrm{~mol} \%$ of DAE is calculated to be $29.7 \mathrm{kcal} / \mathrm{mol}$. The apparent over-all rate of the DAE copolymerization is much slower than that

Table II. Apparent over-ali rate of copolymerition of DAE with styrene

\begin{tabular}{cccc}
\hline \multirow{2}{*}{ Temp, ${ }^{\circ} \mathrm{C}$} & \multicolumn{2}{c}{ Molar ratio } & \multirow{2}{*}{$R_{p}, \mathrm{wt} \% / \mathrm{hr}$} \\
\cline { 2 - 3 } & DAE & Styrene & \\
\hline 60 & 10 & 90 & 0.22 \\
60 & 30 & 70 & 0.60 \\
60 & 50 & 50 & 0.80 \\
60 & 70 & 30 & 0.61 \\
50 & 50 & 50 & 0.12 \\
12 & 50 & 50 & 1.06 \\
\hline
\end{tabular}

a Total monomer concn, $1 \mathrm{~mol} / l$ in benzene; catalyst, $1 \mathrm{~mol} \%$ of azobisobutyronitrile.

Polymer J., Vol. 1, No. 5, 1970 
Alternating Copolymers from trans-1, 2-Dibenzoylethylene and trans-1, 2-Diacetylethylene

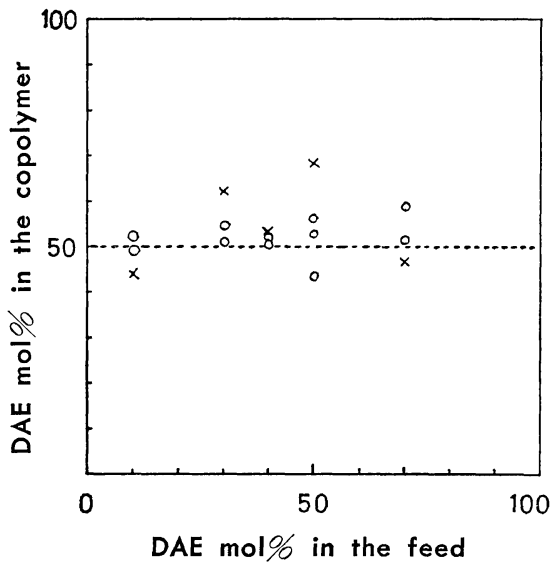

Figure 5. Composions of the copolymers from DAE and styrene; $O$, by elementary analysis; $\times$, by NMR.

of DBE as is clear from a comparison of Table I with Table II.

The copolymer compositions determined either by the elementary analysis or by NMR spectra are shown Figure 5. It may be seen from Figure 5 that the copolymer compositions are scattered near the line of $50 \mathrm{~mol} \%$ of the DAE unit in the copolymer, although the values from the two independent methods do not coincide perfectly since they scattered due to the variation in the unit of the polymer end because of low molecular weight.

\section{Anionic Copolymerization of DBE or DAE}

The anionic copolymerization of DBE or DAE with styrene was carried out in tetrahydrofuran solution at $-78^{\circ} \mathrm{C}$, using $\mathrm{BuLi}$ as an initiator. A red color appeared soon after the addition of BuLi in a nitrogen atmosphere. When the tetrahydrofuran solution was poured in methanol, only polystyrene was obtained and neither DBE nor DAE units were found in the recovered polymer. Therefore, DBE and DAE do not cause the anionic copolymerization with styrene.

\section{Reactivity of $D B E$ and $D A E$}

An $\alpha, \beta$-unsaturated carbonyl compound adds a nucleophilic reagent due to the electrondeficient character of the double bond, and this addition can be used to estimate the character of the double bond of DBE and DAE. The rates of the addition reaction of DBE with $n$ -

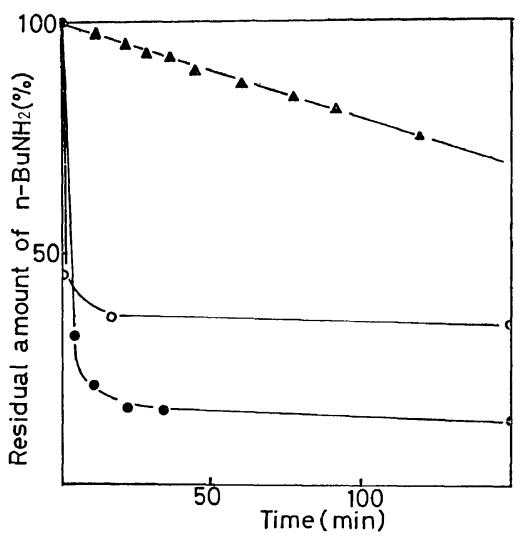

Figure 6. Rate of the addition reaction of DBE or DAE with $n$-butylamine: monomer concn, $1 \mathrm{~mol} / l$ in $\mathrm{THF}, 0^{\circ} \mathrm{C}$; O, DAE; $\bullet$, DBE; $\Delta$, ethyl acrylate.

butylamine at $0^{\circ} \mathrm{C}$ are shown in Figure 6 , where the rates are compared with the addition reaction of ethyl acrylate. The initial rate of the addition reaction of DBE with the amine is faster than that of DAE. However, both rates are much faster than that of ethyl acrylate, indicating that the double bond of DBE and DAE is markedly different in its electrondeficient character from ordinary $\alpha, \beta$-unsaturated carbonyl compounds such as ethyl acrylate.

Neither new absorptions nor even a shift of absorption were observed in the ultraviolet spectra of the mixed solutions of DBE or DAE with styrene or $n$-butyl ether in chloroform, and monotonous increases in optical density were observed by means of the continuous variation method as proposed by Job. Therefore it is not clear whether or not a charge-transfer complex is formed in the monomer solutions.

\section{DISCUSSION}

It was found that both DBE and DAE copolymerized with styrene in the presence of a radical initiator to yield copolymers with constant composition regardless of the monomer ratios in the feed, and that the apparent over-all rate of the DBE copolymerization was faster than that of the DAE copolymerization. Since both DBE and DAE did not homopolymerize at all under the same reaction conditions, it can 
be said that both monomers have a strong tendency to form alternating copolymers with styrene in the presence of a radical initiator. However neither monomer copolymerized with styrene with an anionic initiator.

DBE copolymerized also with $n$-butyl vinyl ether to yield an alternating copolymer with a radical initiator, although the rate was much slower than that with styrene, while DAE did not copolymerize at all with $n$-butyl vinyl ether.

The rate of the addition reaction of DBE with $n$-butylamine was faster than that of DAE and this result suggests that the electron deficient character of DAE might be weaker than that of DBE. The difference in the copolymerization rate between DBE and DAE suggests also that the double bond of DBE has a greater reactivity toward donor monomers than that of DAE, probably because of the inductive effect of two methyl groups on both sides of the carbonyl groups of DAE.

\section{$\mathrm{CH}_{3} \mathrm{COCH}=\mathrm{CHCOCH}_{3}$ (DAE)}

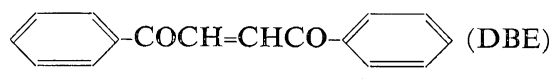

The copolymerization of DAE derivatives revealed that electron-donating or accepting groups on the phenyl group did not have any effect on the alternating tendency of the copolymerization. Since DAE yielded an alternating copolymer with styrene, the effect of alkyl or aromatic groups on both sides of the carbonyl groups does not influence the alternating tendency of $\alpha, \beta$-unsaturated carbonyl compounds, which is primarily under the control of two carbonyl groups on the double bond, and these groups influence only the rate of copolymerization.

Although neither DBE nor DAE apparently form a charge-transfer complex in the monomer solution with donors, the alternating tendency of DBE and DAE might be increased considerably by a strongly favorable polar effect between the electron-deficient DBE or DAE and the electron-rich monomers, possibly even to the point of the point of the formation of a charge-transfer complex in the transition state of the copolymerization as proposed by Walling, ${ }^{8}$ because the apparent over-all rates of the copolymerization reach a maximum at the molar ratio of $50 \mathrm{~mol} \%$ in the monomer feed, except the case of the DBE and styrene system.

The molecular weights of each copolymer were less than 10000 and it was difficult to obtain a high molecular weight copolymer since the monomer concentration in benzene was limited to less than $1 \mathrm{~mol} / l$ because of the low solubility of DBE in benzene.

Neither DBE nor DAE copolymerized with styrene in the presence of an anionic initiator. The DBE or DAE solutions in tetrahydrofuran were colored a deep red in a high vacuum with the addition of sodium naphthalene, indicating that the electron-transfer process had occurred between DBE or DAE and sodium naphthalene. The red color vanished rapidly when the solution was exposed to air and DBE or $\mathrm{BAE}$ were recovered quantitatively from the solution. It is presumed that DBE or DAE may form ketyl anions too stable to initiate the polymerization, possibly because of the conjugation of the two carbonyl groups with the double bond.

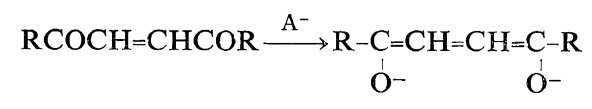

Acknowledgement. The authors wish to thank Dr. K. Nukada of the Toray Co. Ltd. for his measurements of the NMR spectra of the copolymers.

\section{REFERENCES}

1. N. Ogata and K. Nakayama, J. Polym. Sci., Part B, 6, 369 (1968).

2. J. B. Conant, et al., J. Amer. Chem. Soc., 45, 1303 (1923).

3. F. Ramirez and S. Dershuwitz, J. Org. Chem., 22, 41 (1957).

4. D. B. Denny, et al., ibid., 28, 778 (1963).

5. D. B. Denny and L. L. Smith, J. Amer. Chem. Soc., 82, 2396 (1960).

6. K. Sanui and N. Ogata, Bull. Chem. Soc. Japan, 41, 1968 (1968).

7. E. R. Garnett and R. L. Grile, J. Amer. Chem. Soc., 75, 3958 (1953).

8. C. Walling, et al., J. Amer. Chem. Soc., 70, 1537 (1948). 